\title{
DELTA - A Novel Ultra-Low Voltage SEM for Electron Spectroscopic Imaging
}

Rasmus R. Schröder ${ }^{1,3}$, Bernd Schindler ${ }^{2}$, Michael Schnell ${ }^{2}$, Christian Hendrich ${ }^{2}$, Jakob Wensorra ${ }^{2}$, Wen-Shan Zhang ${ }^{1}$, Jörg Eisele ${ }^{3}$, Lisa Veith ${ }^{1}$, Jochen Kammerer ${ }^{3}$, Martin Pfannmöller ${ }^{3}$, Dirk Preikszas ${ }^{2}$, Irene Wacker ${ }^{3}$

1. Cryo Electron Microscopy, BioQuant, Universitätsklinikum Heidelberg, Heidelberg, Germany.

2. Carl Zeiss Microscopy, Oberkochen, Germany.

3. Centre for Advanced Materials (CAM), Universität Heidelberg, Heidelberg, Germany.

In the last few years, low-voltage TEM [1] was instrumental for changing paradigms of imaging using low energy electrons. For very thin and highly conductive samples low-voltage TEM is clearly advantageous, but one faces increasing ionization and thus increased beam damage for less conductive samples [2]. This conundrum can be overcome by further reducing electron energy and using SEM detection mechanisms instead of TEM or STEM.

Based on the DELTA-technology [3] we demonstrate here the possibility of conventional SE and BSE imaging as well as electron spectroscopic imaging (SEM-ESI) at electron landing energies down to 50 $\mathrm{eV}$, well below typical beam damaging energies. Such ultra-low landing energies require aberration correction and the development of new filter and detector technologies if high resolution SEM-ESI needs to be achieved. A novel SEM prototype combining all these technologies has now been developed in the framework of our DELTA-project. The correction of spherical and chromatic aberrations allows even for electrons at ultra-low energies high resolution imaging (better than $1 \mathrm{~nm}$ at $50 \mathrm{eV}$ ). As less beam damage is observed we utilize the increased applicable electron dose for electron spectroscopy. Here we record either spectra of SE to obtain "work function / electron affinity contrast" [4,5], or spectra of BSE. The DELTA-SEM facilitates ESI in a similar way to energy loss imaging and spectroscopy in TEM.

Fig. 1 shows the current DELTA-SEM prototype with its complex electron optics made possible by the Delta-shaped beam splitter and the electrostatic electron mirror. This SEM allows energy-filtered imaging (Fig. 2) using an electrostatic energy filter combined with a scintillator electron detector (DELTADetector $^{\circledR}$ design). Electrons of different energy range contribute selectively to the final image when the electric field in the DELTA-detector is varied. Fig. 3 shows an example of imaging and SE spectroscopy of semiconducting organic materials. Their SE intensity, compared to the gold substrate, and their ESI spectra correlate with the relative conductivity of the materials.

Even more interesting than spectral imaging with SEs is the use of BSEs. While SE signals are higher than BSE signals at conventional landing energies, BSE gains on SE at very low energies. Fig. 4 shows the typical intensity profiles for BSE and SE of $\mathrm{Al}$ at $100 \mathrm{eV}$ and $500 \mathrm{eV}$ landing energies. Thus, at lower energies the BSE signal can be studied in detail: The zero-loss peak as the signal of immediate backscattering is trivial, whereas the spectrum at low energy loss can be interpreted as multiple elastic inelastic scattering. For Al the excitation of the surface plasmon can be identified (cf. Fig. 4).

At present we work on the deconvolution of the energy spread function of the DELTA-SEM [5] and use landing energies down to $50 \mathrm{eV}$ for spectral imaging of organic materials. Model simulations and experiments on the bulk heterojunction of organic solar cells [6] indicate the possibility to distinguish different materials and to utilize SEM-ESI for functional imaging of highly beam sensitive materials. 
References:

[1] U Kaiser et al., Ultramicroscopy 111 (2011) p. 1239.

[2] E Majorovits et al., Biophysical J 110 (2016) p. 776.

[3] M Steigerwald et al., Frontiers of Characterization and Metrology for Nanoelectronics (2013).

[4] J Cazaux, Ultramicroscopy 110 (2011) p. 242.

[5] cf. J Eisele et al, Microscopy and Microanalysis Conference Proceedings of this conference.

[6] cf. J Kammerer et al., Microscopy and Microanalysis Conference Proceedings of this conference.

[7] The authors acknowledge funding of the DELTA project by the German Federal Ministry of Education and Research to RRS (FKZ: 13GW0044).

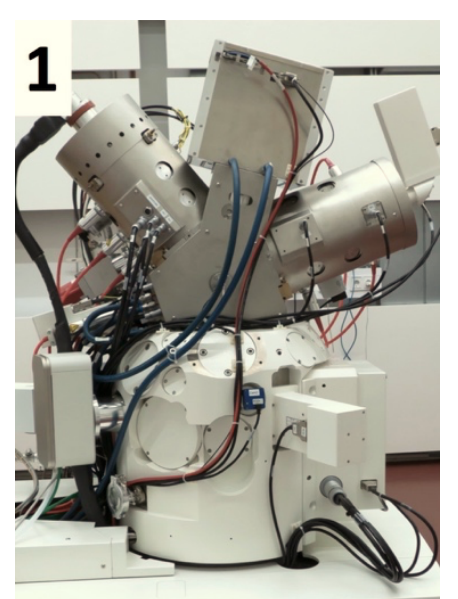

Figure 1. DELTASEM, visible are the electron gun (top left), the aberration corrector (middle right) and the deltashaped beam splitter (centre, above the sample chamber).

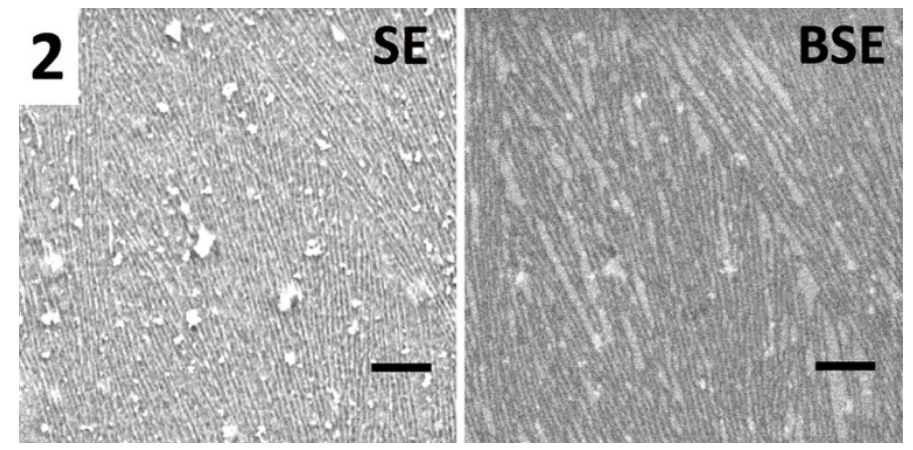

Figure 2. Energy-filtered secondary electron (SE) and backscattered electron (BSE) images of uncoated polymer fibers (about $10 \mathrm{~nm}$ in diameter) on silicon wafer at $500 \mathrm{eV}$ landing energy. Scale bar $200 \mathrm{~nm}$.
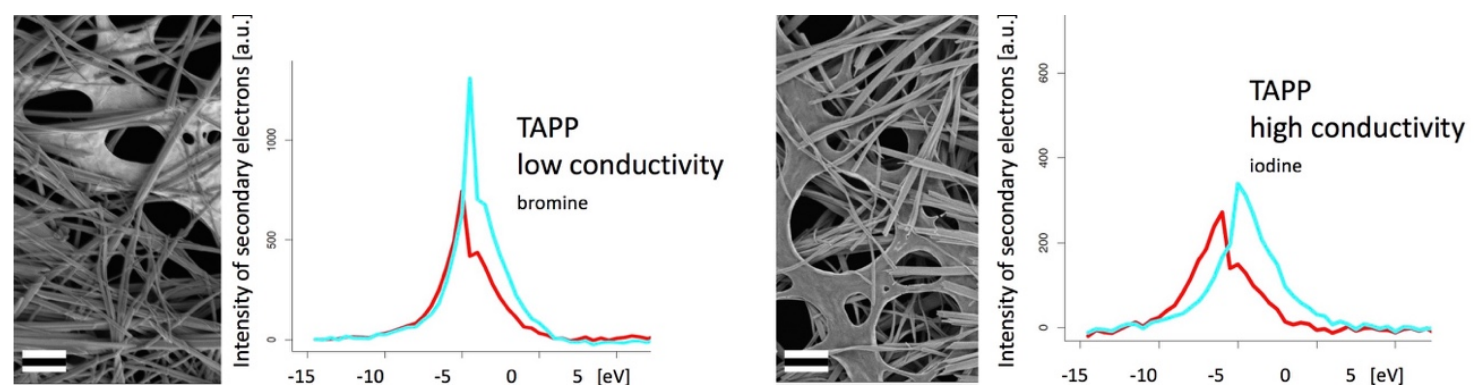

Figure 3. SE images and spectra of nano-crystals of halogen substituted tetraazaperopyrene (TAPP) on gold coated holey carbon film. Note the correspondence of SE intensity relative to the conducting gold film (cyan) with expected charge mobility of the material. Landing energy $1 \mathrm{keV}$. Scale bar $1 \mu \mathrm{m}$.
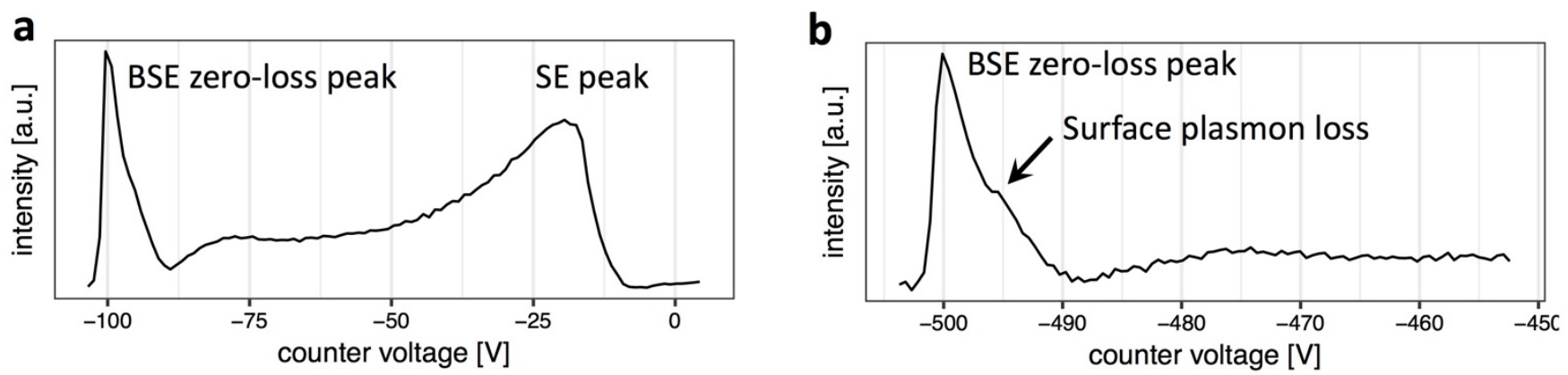

Figure 4. Electron spectra of $\mathrm{Al}$ at (a) $100 \mathrm{eV}$ and (b) $500 \mathrm{eV}$ landing energy. Note the relative intensity of SE and BSE peaks at ultra-low landing energies. As mainly the surface of the Al sample is probed BSE carry the signal of the Al-surface plasmon instead of the usual volume plasmon peak in TEM-EEL spectra. 